\title{
Influence of the contact model on the dynamic response of the human knee joint
}

\author{
M Machado $^{1 *}$ P Flores $^{1}$, J Ambrosio $^{2}$, and A Completo ${ }^{3}$ \\ ${ }^{1}$ Centro de Tecnologias Mecânicas e de Materiais (CT2M), Departamento de Engenharia Mecânica, Universidade do \\ Minho, Guimaraes, Portugal \\ ${ }^{2}$ Instituto de Engenharia Mecânica (IDMEC), Instituto Superior Técnico, Universidade Técnica de Lisboa, \\ Lisboa, Portugal \\ ${ }^{3}$ Centro de Tecnologia Mecânica e Automação (TEMA), Departamento de Engenharia Mecânica, Universidade de \\ Aveiro, Aveiro, Portugal
}

The manuscript was received on 29 November 2010 and was accepted after revision for publication on 26 May 2011.

DOI: $10.1177 / 1464419311413988$

\begin{abstract}
The goal of this work is to study the influence of the contact force model, contact geometry, and contact material properties on the dynamic response of a human knee joint model. For this purpose, a multibody knee model composed by two rigid bodies, the femur and the tibia, and four non-linear spring elements that represent the main knee ligaments, is considered. The contact force models used were the Hertz, the Hunt-Crossley, and the Lankarani-Nikravesh approaches. Results obtained from computational simulations show that Hertz law is less suitable to describe the dynamic response of the cartilage contact, because this pure elastic model does not account for the viscoelastic nature of the human articulations. Since knee can exhibit conformal and non-conformal contact scenarios, three different geometrical configurations for femur-tibia contact are considered, that is convex-convex sphere contact, convex-concave sphere contact, and convex sphere-plane contact. The highest level of contact forces is obtained for the case of convex-convex sphere contact. As far as the influence of the material contact properties is concerned, the dynamic response of a healthy and natural knee is analysed and compared with three pathological and two artificial knee models. The obtained results demonstrate that the presence of the cartilage reduces significantly the knee contact forces.
\end{abstract}

Keywords: knee joint, contact model, contact geometry, contact material, multibody dynamics

\section{INTRODUCTION}

It is known that in contact mechanics problems, the evaluation of the contact pressures and stresses that develop between the contacting bodies depends on the constitutive law used. This law typically depends on the material properties of the contacting surfaces and on the contact distance, which determines

*Corresponding author: Centro de Tecnologias Mecânicas e de Materiais (CT2M), Departamento de Engenharia Mecânica, Universidade do Minho, Campus Azurem, 4800-058 Guimaraes, Portugal.

email:margarida@dem.uminho.pt whether the bodies are in contact or not. In order to compute the contact distance, the position, orientation, and contact geometry of potential contact bodies have to be known. In short, three main ingredients have to be considered to perform a dynamic contact-impact analysis, namely the definition of the contact geometry, the contact detection approach, and the application of the constitutive contact force law [1-3].

Analytical functions of regular shapes, such as planes, spheres, ellipsoids, among others, are, in general, the best choice to describe simple contact geometries [4]. When the contact surfaces present complex configurations, more sophisticated fitting 
approaches must be considered to obtain an accurate surface representation [5, 6]. However, the advanced fitting techniques take too much computational time, which could penalize the global efficiency of the method. This is the reason why some authors split complex geometries using multiple regular shapes $[7,8]$.

There are some collision detection methods developed for computer graphics applications such as axisaligned bounding box trees or oriented bounding box trees that have been widely used to increase the performance of contact detection algorithms [9-11]. These bounding volume hierarchies are refined methods based on polygonal meshes and are available in software packages such as RAPID, I-COLLIDE, V-COLLIDE, SOLID, and V-Clip. In general, these approaches are computationally costly and can only be applied to the case of convex contact geometries [10]. The conformality of the contact geometries plays a key role in the contact detection approach. Recently, Choi et al. [11] developed a new collision detection method called triangle soup average plane contact [11], which is quite robust, efficient, and can be applied to convex and concave geometries, without the need of using any graphics hardware.

In a simple manner, it can be stated that the contact force laws are functions on the distance between the potential contact points and on the material properties $[\mathbf{1}-\mathbf{3}, \mathbf{1 2}, \mathbf{1 3}]$. Additionally, the contact materials present a non-linear damping behaviour that should be taken into account by the constitutive contact law $[\mathbf{1}-\mathbf{3}, \mathbf{1 3}]$. Friction and lubrication effects are also important phenomena that can significantly affect the contact responses [14-16]. In applications, such as wear analysis, the contact area and pressure distribution have to be determined and, hence, a contact law must be suitable to accommodate their demands $[17,18]$. Furthermore, some contact materials are multilayer and, consequently, the contact law has to be able to compute the stresses at the surface as well as at the sub-surfaces. The computational efficiency associated with the contact law is an important issue in the context of multibody dynamics involving contact-impact events, because besides the evaluation of the contact forces themselves, in general, the contact procedure also demands the computation of other state variables, such as the indentation and the contact velocity. Furthermore, the contact force law should also contribute to a stable and efficient numerical resolution of the equations of motion [3, 19].

In this article, the knee joint contact problem is studied. This is a complex and an important contact problem, in the measure that some of the most common knee injuries and diseases, such as osteoarthritis (OA), ligamentous rupture, and meniscal tear, can be significantly be affected by intensive and abnormal contact interactions [20]. When initiated, these pathologies induce anomalous and more aggressive contact loads which results in non-physiologic gait patterns and local pain that could rapidly lead to a knee arthroplasty, i.e. to a joint replacement. This is a challenging topic since there is no a standard non-invasive approach to measure in vivo knee loads, which means that the knee contact patterns and pressures have to be predicted by computational methods [2]. Wismans [21] was one of the first researchers who evaluated the knee contact forces. In this study, the knee contact surfaces were modelled using polynomial functions and the contact forces were evaluated using a linear elastic contact model, considering a non-conformal contact scenario. Moeinzadeh [22], Engin and Tumer [23], and Ling et al. [24] developed a dynamic two-dimensional model of the knee joint based on similar assumptions. An analogous planar model was developed by Abdel-Rahman and Hefzy [25], which was later extended to three dimensions in reference [26]. Hirokawa [27] applied the pure elastic Hertz law to compute the contact forces at the patellofemoral joint. Blankevoort et al. [28] introduced a new concept of articular contact based on the simplified theory of contact developed by Kalker [29] for thin layers of isotropic and linear-elastic material bonded to a rigid foundation. Blankevoort and Huiskes [30] extended this work to the threedimensional (3D) case.

Gill and O'Connor [31] and Chittajallu and Kohrt [32] used four-bar linkages to model the knee joint, where the tibia is the ground, the femur the coupler, and the ligaments represented by the remaining links. In these works, the interpenetration between the contacting bodies is not allowed, and so, despite the contact points are determined, the contact forces are not evaluated. In sharp contrast, other researchers applied advances techniques, such as finite element (FE) methods, to compute the contact forces at the knee joint [33-35]. Li et al. [36] performed a comparative study, where the pressure distribution along the contact surface of an articulating joint model was analysed using different numerical and analytical methods, namely the discrete rigid element method, the FE method, a simplified elasticity solution, and a modified Hertzian theory. Pandy et al. [37] developed a complete knee joint model that accounts for contact forces, together with the four ligaments and 13 muscles. In this work, the interpenetration of the femur and the tibia is by considering the modelling of the cartilage as a thin, linear, and elastic layer assembled in rigid bone, being the elastic foundation model used to evaluate the contact forces [37]. 
Zhu et al. [38] modelled the geometry of the tibiofemoral joint as two elliptic curved surfaces and use the Hertz elastic contact law to calculate the contact stresses and pressures. Kwak et al. [39] represented the knee articular surfaces by parametric surface equations and applied the rigid foundation model to compute the contact forces. Piazza and Delp [40] presented a rigid body dynamic model of a total knee replacement (TKR) by performing a step-up task. In this model, the articulating surfaces of the femoral, tibial, and patellar components are represented by 3D polyhedral meshes and the contact detection is performed using RAPID library. Piazza and Delp [40] considered the knee contact problem as a linear complementarity problem. Li et al. [41] studied the influence of the cartilage thickness and the cartilage material properties on the contact pressures and stresses using five FE models. Dhaler and Kahn [42] applied basis functions to describe the geometry of the articular surfaces and used a Hertzian contact law to evaluate the patellofemoral contact forces. Caruntu and Hefzy [43] updated the model published by Abdel-Rahman and Hefzy [26] including the patellofemoral joint. Instead of modelling the articular surfaces as spheres and planes, Coons' bicubic surface patches are used. Bei and Fregly [44] developed a 3D dynamic model of the knee joint. The articular surfaces are modelled using non-uniform rational basis spline (NURBS) surfaces and the contact forces are evaluated using elastic foundation model. These authors also proposed two different contact detection approaches, namely minimum distance and ray firing, which can be combined to ensure an accurate detection of the contact points, especially in conformal contact scenarios [44]. Besier et al. [45] and Fernandez and Hunter [46] used the FE method to calculate contact stresses and strains at the patellofemoral joint. Han et al. [47] proposed a 3D FE knee model, where the articular cartilage is considered to be biphasic: the solid phase is assumed to be linearly elastic and incompressible, and the fluid phase is taken as incompressible, non-viscous, and with indentation-dependent permeability.

It is known that rigid body models with contact based on elastic foundation theories require significantly less computational time than corresponding deformable FE methods. However, Halloran et al. [48] affirm that potential differences in predicted kinematics between these models were not well understood and, so, that it was unclear if the estimates of contact area and pressure are acceptable. Thus, in their study, rigid elastic foundation and deformable FE models of tibiofemoral contact were developed and the predicted kinematics and contact mechanics from both representations during gait loading conditions compared using three different implant designs [48]. Koo and Andriacchi [49] developed a comparative study to evaluate the influence of the global functional loads and the local contact anatomy on articular cartilage thickness at the knee. In this study, the articular surfaces are modelled as two semi-ellipsoids and the medial/lateral contact pressure ratio was calculated using elliptical Hertzian contact stress theory. The results reported by Koo and Andriacchi [49] revealed that contact pressure is higher in the lateral than medial compartments and cartilage thicker in the lateral than medial compartments. Pérez-González et al. [19] realize that different models have been used in the literature for the simulation of surface contact in biomechanical knee models; however, there is a lack of systematic comparisons of the models applied to simulate a common case, which will provide relevant information about their accuracy and suitability for application in models of the artificial knee. Therefore, these authors performed a comparative study using the Hertz model, the elastic foundation model, and the FE model to evaluate the contact forces at an artificial knee [19]. Lin et al. [50] presented a novel surrogate modelling approach to perform computational efficient 3D elastic contact analyses within the multibody dynamic simulations.

In the present planar work, a knee joint model is presented and the influence of the contact model on its dynamic response is assessed. In this comparative study, not only the contact force law is evaluated, but also the contact geometry and material properties are used as variables. Hertz [51], Hunt and Crossley [52], and Lankarani and Nikravesh [53] force models are compared for equivalent contact conditions. Regarding the contact geometry, since the tibial plateaus do not exhibit the same conformality in both knee compartments, three contact scenarios are tested, namely convex-convex contact, convexconcave contact, and convex sphere-plane contact. In order to examine healthy, pathological, and artificial knee response to the same contact loads, the contact material properties and surfaces thickness are also considered as variables. Numerical simulations are performed using a computational code called MUBODYNA developed under the framework of multibody system (MBS) methodologies $[\mathbf{5 4}, \mathbf{5 5}]$ to perform efficient dynamic analysis of general MBS systems [56, 57].

\section{KNEE CONTACT MODELLING}

This section deals with the fundamental issues of the mathematical human knee model used in this study, which has been developed under the framework of 


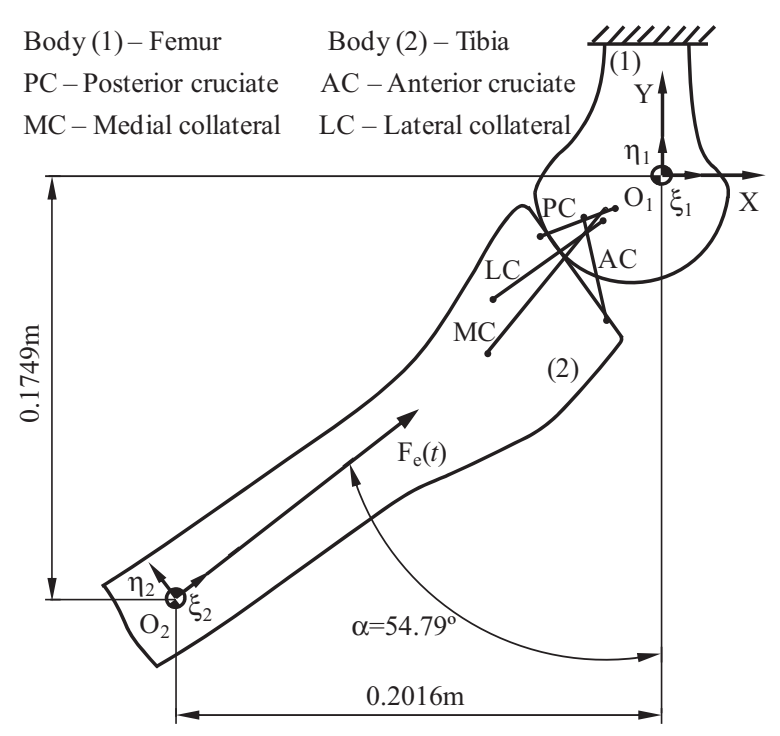

Fig. 1 Initial configuration of the multibody knee joint model

MBS methodologies [2]. The knee model is composed by two rigid bodies, the tibia and the femur, which describe a general planar motion in the sagittal plane. The femur is considered to be stationary, while the tibia does not have any kinematic constraint. The tibia is connected to the femur by four knee ligaments, namely the anterior cruciate (AC) ligament, the posterior cruciate (PC) ligament, the medial collateral (MC) ligament, and the lateral collateral (LC) ligament. These ligaments are modelled as non-linear spring elements. The following force-elongation mathematical relation is utilized for each ligament [21]

$$
F_{1}= \begin{cases}k_{1}\left(l_{1}-l_{1}^{0}\right)^{2} & \text { if } l_{1}>l_{1}^{0} \\ 0 & \text { if } l_{1} \leq l_{1}^{0}\end{cases}
$$

where $k_{1}$ is the ligament stiffness and $l_{1}$ and $l_{1}^{0}$ the current and the unstrained lengths of the ligaments, respectively.

Figure 1 shows two bodies $i$ and $j$ that represent the tibia and femur, respectively. Body-fixed coordinate systems $\xi \eta$ are attached to each body, while $X Y$-coordinate frame represents the global coordinate system. The origin of the femur coordinate system is located at the intercondylar notch and is coincident with the global coordinate axis. The origin of the tibia coordinate system is located at the centre of mass of the tibia, with the local $\xi$-axes directed proximally and $\eta$-axes directed posteriorly. The absolute rotation angles of the local coordinate systems of bodies $i$ and $j$ are denoted by $\phi_{i}$ and $\phi_{j}$, respectively. The Cartesian coordinates of centres of mass and inertia properties of the femur and tibia used in this study
Table 1 Local coordinates of the insertion points and physical properties of the ligaments $[\mathbf{2 1}, \mathbf{2 2}]$

\begin{tabular}{lrrrr}
\hline Ligament & \multicolumn{1}{c}{ AC } & \multicolumn{1}{c}{ PC } & \multicolumn{1}{c}{ MC } & \multicolumn{1}{c}{ LC } \\
\hline$\xi_{\mathrm{f}}^{l}(\mathrm{~m})$ & -0.0330 & -0.0190 & -0.0230 & -0.0250 \\
$\eta_{\mathrm{f}}^{1}(\mathrm{~m})$ & -0.0170 & -0.0140 & -0.0140 & -0.0190 \\
$\xi_{\mathrm{t}}^{\mathrm{l}}(\mathrm{m})$ & 0.2130 & 0.2100 & 0.1630 & 0.1780 \\
$\eta_{\mathrm{t}}(\mathrm{m})$ & -0.0090 & 0.0350 & 0.0080 & 0.0250 \\
$l_{1}^{0}(\mathrm{~m})$ & 0.0438 & 0.0332 & 0.0784 & 0.0562 \\
$k_{\mathrm{l}}\left(\mathrm{kN} / \mathrm{m}^{2}\right)$ & 35000 & 30000 & 15000 & 15000 \\
\hline
\end{tabular}

correspond to a male subject of weight $76 \mathrm{~kg}$ and height $1.8 \mathrm{~m}$ [2].

The unstrained lengths of the four ligaments are adopted from the Moeinzadeh's [22] work. The initial position of the tibia at $54.79^{\circ}$ of knee flexion is elected because it corresponds to a particular position where the ligaments are in a relatively relaxed condition, and therefore the knee contact forces can be neglected. The local coordinates of the ligament insertion points, as well as their physical properties (unstrained length and stiffness) are listed in Table 1.

Since the knee kinematics is not prescribed, a force constraint has to be included to the system in order to avoid the separation of the tibia due gravitational action. Thus, an external force is applied at the centre of mass of the tibia directed proximally, as illustrated in Fig. 1. The aim of this force is to promote the tibiofemoral contact and also to provide the knee motion on the anterior-posterior direction, from an initial position of $54.79^{\circ}$ of flexion to a final position of $0^{\circ}$ of extension. The hyperextension scenario has not been taken into account in the computational simulations, although it is important to mention that, in general, $1-3^{\circ}$ of hyperextension is anatomically tolerable, beyond which joint failure becomes unavoidable. The external applied force, $F_{\mathrm{e}}$, is expressed as

$$
F_{\mathrm{e}}=A \mathrm{e}^{-4.73\left(\frac{t}{t_{d}}\right)^{2}} \sin \left(\frac{\pi t}{t_{\mathrm{d}}}\right)
$$

which is an exponentially decaying sinusoidal pulsed function with a duration $t_{\mathrm{d}}$ and an amplitude $A$ [22]. The same type of applied external force has been used in computational simulations of other biomechanical models, such as in modelling and simulation of the force of the quadricep muscle group in knee extension and of human head neck studies $[\mathbf{2 2}, \mathbf{2 6}, \mathbf{5 8}]$.

\subsection{Mathematical contact force models}

In a broad sense, the different methods to solve the contact-impact problem in multibody dynamics are either continuous or discontinuous approaches. Within the continuous approach, the methods 
commonly used are the continuous force models and the unilateral constraint methodology, based on the complementarity formulation $[\mathbf{1}, \mathbf{3}]$. The continuous contact force models, also known as penalty or compliant approaches, represent the forces arising from collisions and assume that the forces and deformations vary in a continuous manner. In these methods, when contact between the bodies is detected, a contact force perpendicular to the plane of collision is applied. The penalty formulations can be understood as if each contact region of the colliding bodies is covered with some spring-damper elements scattered over their surfaces. Alternatively, the complementarity formulations resolve the contact dynamics problem using the unilateral constraints to compute contact impulses or forces to prevent penetration from occurring. Thus, when contact is detected, a kinematic constraint is introduced in the system's equations of motion, that is maintained while the reaction forces are compressive, and removed when the impacting bodies rebound from contact $[\mathbf{1}, \mathbf{3}]$. As far as the discontinuous approach is concerned, it is assumed that the impact occurs instantaneously and the resolution of the equations of motion is halted at the time of impact. Then, a momentum balance is performed to calculate the post-impact velocities of the bodies involved in the contact. The resolution is then resumed with the updated velocities until the next impact occurs. In the discontinuous method, the dynamic analysis of the system is divided into two intervals, before and after impact. The discontinuous approach is relatively efficient, but the unknown duration of the impact limits its application because for long contacts, the system configuration can change significantly.

The continuous contact force models have been gaining significant importance in the context of MBSs with contacts; thanks to their computational simplicity and efficiency. In particular, the contact forces developed at the knee joint have been evaluated using compliant approaches $[\mathbf{1 9}, \mathbf{4 4}]$. In this study, three different continuous contact force models are used to evaluate the normal contact forces developed at the knee joint, namely the Hertz contact law, the Hunt and Crossley model, and Lankarani and Nikravesh formulation. The Hertz law is a very popular and well-known contact force model that relies on the elasticity theory principles and considers five general assumptions: (a) the contact area is elliptical, (b) each body is approximated by an elastic half-space loaded over the plane elliptical contact area, (c) the size of the contact area must be small compared to the size of each body and to the radii of curvature of the surfaces, (d) the strains are sufficiently small for linear elasticity to be valid, and (e) the contact is frictionless $[\mathbf{5 9}, \mathbf{6 0}]$. The Hertz contact law relates the contact force with a non-linear power function of deformation and can be explicitly written in the following form

$$
F_{\mathrm{N}}=K \delta^{n}
$$

where $F_{\mathrm{N}}$ is the normal contact force, $K$ the generalized stiffness parameter, and $\delta$ the relative indentation. The exponent $n$ is typically equal to 1.5. In turn, the generalized stiffness parameter depends on the geometric and material properties of the contacting bodies. For convex-convex sphere contacts, convexconcave sphere contacts, and convex sphere-plane contacts, the generalized stiffness parameter can be evaluated as, respectively

$$
\begin{aligned}
& K=\frac{4}{3\left(\frac{1-v_{\mathrm{f}}^{2}}{E_{\mathrm{f}}}+\frac{1-v_{\mathrm{t}}^{2}}{E_{\mathrm{t}}}\right)} \sqrt{\frac{R_{\mathrm{f}} R_{\mathrm{t}}}{R_{\mathrm{f}}+R_{\mathrm{t}}}} \\
& K=\frac{4}{3\left(\frac{1-v_{\mathrm{f}}^{2}}{E_{\mathrm{f}}}+\frac{1-v_{\mathrm{t}}^{2}}{E_{\mathrm{t}}}\right)} \sqrt{\frac{R_{\mathrm{f}} R_{\mathrm{t}}}{R_{\mathrm{f}}-R_{\mathrm{t}}}} \\
& K=\frac{4}{3\left(\frac{1-v_{\mathrm{f}}^{2}}{E_{\mathrm{f}}}+\frac{1-v_{\mathrm{t}}^{2}}{E_{\mathrm{t}}}\right)} \sqrt{R_{\mathrm{f}}}
\end{aligned}
$$

where $v_{\mathrm{f}}$ and $v_{\mathrm{t}}$ are the Poisson's ratios of femur and tibia, $E_{\mathrm{f}}$ and $E_{\mathrm{t}}$ the Young's moduli of femur and tibia, and $R_{\mathrm{f}}$ and $R_{\mathrm{t}}$ the radii of femur and tibia, respectively.

The Hertz contact law is a purely elastic model in nature and it does not include any energy dissipation associated with the contact process. In fact, the energy transferred and dissipated in a contact event are quite complex phenomena that have motivated some researchers to extend the Hertz theory in order to accommodate the loss of energy during contact events. Hunt and Crossley [52] and Lankarani and Nikravesh [53] are among the few authors who extended the Hertz law to include some energy loss due to internal damping. They wrote the normal contact force as

$$
F_{\mathrm{N}}=K \delta^{n}+D \dot{\delta}
$$

where the first term corresponds to the elastic force and the second term the energy dissipated during the contact. In equation (7), the quantity $D$ is the damping coefficient and $\dot{\delta}$ the relative normal contact velocity. The damping coefficient can be written as

$$
D=\chi \delta^{n}
$$

In turn, the hysteresis factor $\chi$ has different expressions for Hunt-Crossley and Lankarani-Nikravesh models, which can be expressed as, respectively 


$$
\begin{aligned}
& \chi=\frac{3 K\left(1-c_{\mathrm{r}}\right)}{2 \dot{\delta}^{0}} \\
& \chi=\frac{3 K\left(1-c_{\mathrm{r}}^{2}\right)}{4 \dot{\delta}^{0}}
\end{aligned}
$$

in which $c_{\mathrm{r}}$ represents the restitution coefficient and $\dot{\delta}^{0}$ the initial relative normal contact velocity, having the remaining parameters with the same meaning as described above.

\subsection{Knee contact geometrical conformality}

The human knee joint presents a complex geometry that can be described with some accuracy using sophisticated mathematical representations such as NURBS surfaces [44]. However, due to its simplicity and efficiency, in this study, the articular geometry of the knee joint is modelled using analytical functions of regular shapes, such as planes and spheres.

Despite the contact geometry has been simplified, the original conformality of the articular knee surfaces is kept, because this factor plays a role of paramount importance in the knee contact problem, particularly in what concerns with the contact detection process. For instance, Fig. 2 depicts two bodies, wherein the location of the centres of mass is the same. In the case of Fig. 2(a), the bodies are not in contact due to their conformal contact nature. In sharp contrast, in Fig. 2(b), the bodies are in contact and have a relative indentation, $\delta$, since the contact is non-conformal.

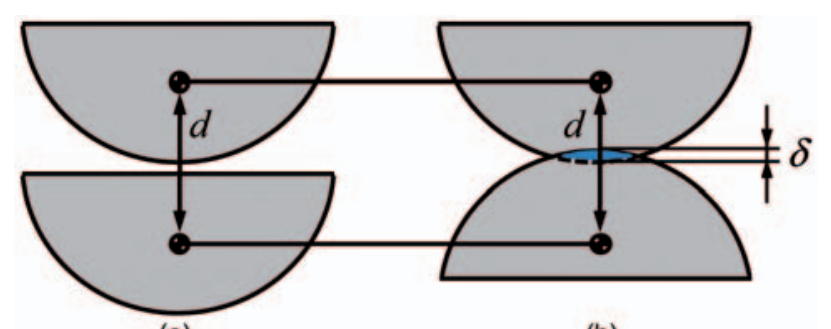

(a)

(b)

Fig. 2 Schematic representation of two contact scenarios: (a) conformal and (b) non-conformal
According to Koo and Andriacchi [49], the femoral condyles present convex curvatures in medial and lateral compartments and the tibial plateaus have concave curvatures in medial compartment and convex curvature in lateral compartment. Based on these observations, three contact scenarios for the knee joint are considered, as illustrated in Fig. 3.

In the first contact scenario, represented in Fig. $3(\mathrm{a})$, both the femoral condyle and the tibial plateau are fitted to convex spheres. This scenario corresponds to the conformality of the knee at lateral compartment. In the second contact scenario, which corresponds to the knee medial compartment, the femoral condyle assumes a convex spherical configuration, and the tibial plateau is modelled as a concave sphere, as shown in Fig. 3(b). The last contact scenario, illustrated in Fig. 3(c), describes the conformality of the knee at the intercondylar notch. In this case, the femur exhibits a convex spherical shape and the tibia is considered a flat surface. The values of the adopted radii for femur and tibia as well as the contact stiffness for each contact scenario are listed in Table $2[499,62]$.

Since three distinct geometrical models are considered, different methodologies to deal with the contact detection have to be applied. Figure 4 shows a schematic representation of each contact scenario, where some scalar and vector information necessary for the contact detection algorithm are pointed out.

In what concerns to the convex-convex sphere model and the convex-concave sphere model of Figs 4(a) and (b), the first step consists of determining the vector $\mathbf{d}$ that connects the centres of the two contact spheres. The vector $\mathbf{d}$ for the convex-convex sphere model and the convex-concave sphere model is, respectively, expressed as

$$
\begin{aligned}
& \mathbf{d}=\mathbf{r}_{j}^{C_{j}}-\mathbf{r}_{i}^{C_{i}} \\
& \mathbf{d}=\mathbf{r}_{i}^{C_{i}}-\mathbf{r}_{j}^{C_{j}}
\end{aligned}
$$

where $\mathbf{r}_{i}^{C_{i}}$ and $\mathbf{r}_{j}^{C_{j}}$ are the global coordinate vectors of centre points $C_{i}$ and $C_{j}$. For both models, the magnitude of the vector $\mathbf{d}$ can be computed as (a)

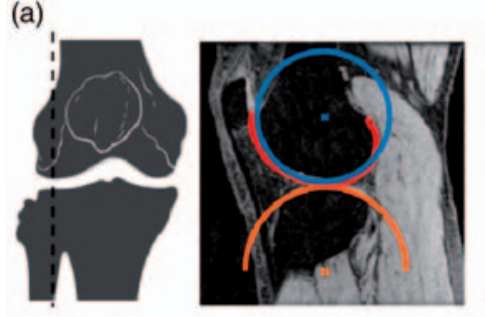

(b)

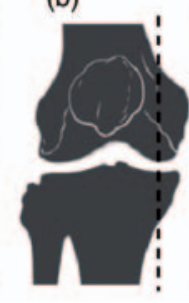

(c)

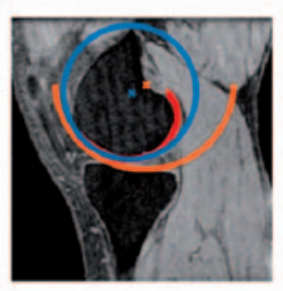

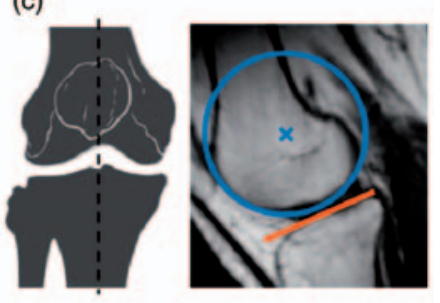

Fig. 3 Images of knee magnetic resonance imaging: (a) lateral, (b) medial, and (c) intercondylar views (adapted from reference [61]) 


$$
d=\sqrt{\mathbf{d}^{T} \mathbf{d}}
$$

To check if the bodies are in contact or not, it is necessary to evaluate the indentation condition. For the contact type in which the femoral condyle and the tibial plateau are considered as convex spheres (Fig. $4(\mathrm{a})$ ), the indentation condition is expressed as

$$
\delta= \begin{cases}0 & \text { if } d>R_{i} \\ \left(R_{i}+R_{j}\right)-d & \text { if } d \leq\left(R_{i}+R_{j}\right)\end{cases}
$$

For the contact between a spherical convex femoral condyle and a spherical concave tibial plateau (Fig. 4(b)), the indentation condition is given by

$$
\delta= \begin{cases}0 & \text { if } d<\left(R_{j}-R_{i}\right) \\ d-\left(R_{j}-R_{i}\right) & \text { if } d \geq\left(R_{j}-R_{i}\right)\end{cases}
$$

Regarding the contact between a spherical femoral condyle and a planar tibial plateau (Fig. 4(c)), the first step of contact detection procedure consists of evaluation of the minimal distance between the bodies. Thus, since the location of the points $A_{j}, B_{j}$, and $C_{i}$ is

Table 2 Femur $\left(R_{\mathrm{f}}\right)$ and tibia $\left(R_{\mathrm{t}}\right)$ radii as well as the contact stiffness parameter $(K)$ used in each contact scenario, namely convex-convex spheres, convex-concave spheres, and convex sphere-plane $[49,61]$

\begin{tabular}{llll}
\hline & $\begin{array}{l}\text { Convex-convex } \\
\text { spheres }\end{array}$ & $\begin{array}{l}\text { Convex-concave } \\
\text { spheres }\end{array}$ & $\begin{array}{l}\text { Convex } \\
\text { sphere-plane }\end{array}$ \\
\hline$R_{\mathrm{f}}(\mathrm{mm})$ & 26.40 & 30.40 & 30.40 \\
$R_{\mathrm{t}}(\mathrm{mm})$ & 36.02 & 75.00 & $\infty$ \\
$K\left(\mathrm{~N} / \mathrm{m}^{1.5)}\right.$ & 2750403 & 5879434 & 3260513 \\
\hline
\end{tabular}

known, the vectors $\mathbf{A C}$ and $\mathbf{A B}$ can be defined and its dot product calculated by

$$
\begin{aligned}
& \mathbf{A C} \cdot \mathbf{A B}=\mathrm{AC}_{x} \mathrm{AB}_{x}+\mathrm{AC}_{y} \mathrm{AB}_{y} \\
& \mathbf{A C} \cdot \mathbf{A B}=\|\mathbf{A C}\| \cdot\|\mathbf{A B}\| \cdot \cos \theta_{A}
\end{aligned}
$$

Based on equations (16) and (17), it is possible to compute the angle between the two vectors, $\theta_{A}$, which helps in the calculation of the distance $d$ between the plane and the centre of the sphere

$$
d=\|\mathbf{A C}\| \cdot \sin \theta_{A}
$$

The last step is to check if the bodies are in contact by evaluating the indentation $\delta$ by

$$
\delta= \begin{cases}0 & \text { if } d>R_{i} \\ R_{i}-d & \text { if } d \leq R_{i}\end{cases}
$$

\subsection{Knee contact material properties}

The mechanical properties of the materials that compose the contacting bodies play a crucial role on the dynamic response of the knee model, because they directly affect the magnitude of the contact forces, the amount of energy dissipated during the contact and, consequently, the motion of the bodies.

The contact material between the femur and the tibia, in a healthy natural knee articulation, is composed by a hyaline cartilage layer with approximated $4.15 \mathrm{~mm}$ of thickness $(2.45 \mathrm{~mm}$ on distal femur and $1.70 \mathrm{~mm}$ on proximal tibia $[\mathbf{6 1}, \mathbf{6 2}])$. However, in several cases, the subject can present a knee pathology that significantly changes the contact material
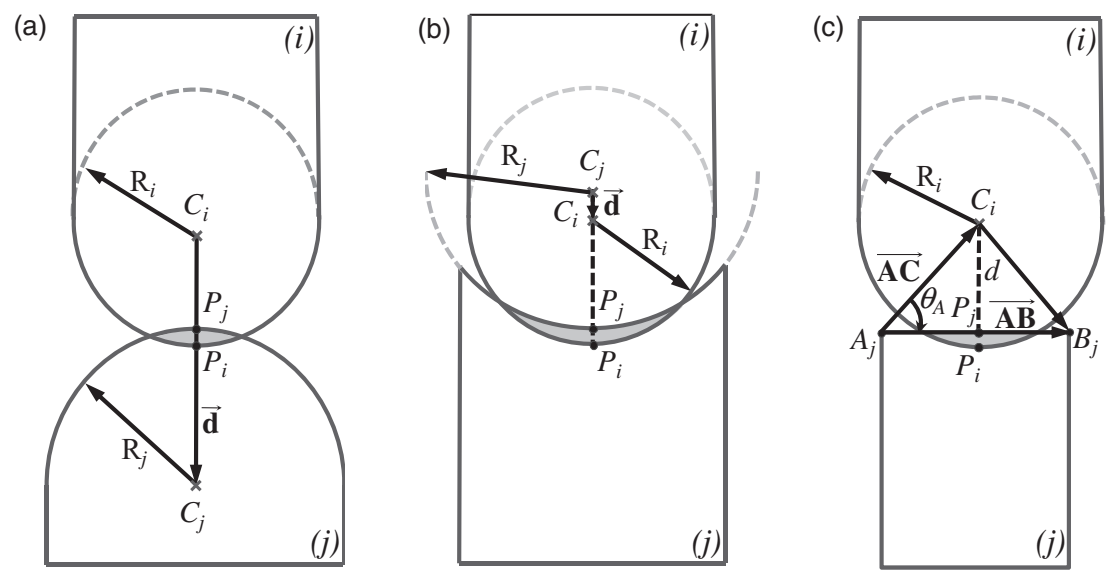

Fig. 4 Schematic representation of the different contact scenarios: (a) convex-convex spheres, (b) convex-concave spheres, and (c) convex sphere-plane 
Table 3 Femur and tibia contact materials considered for each knee contact model studied

\begin{tabular}{|c|c|c|c|}
\hline Knee & Femur material & Tibia material & Stiffness $\left(\mathrm{N} / \mathrm{m}^{1.5}\right)$ \\
\hline Healthy (SL) & Hyaline cartilage & Hyaline cartilage & $3.26 \mathrm{E}-06$ \\
\hline \multirow[t]{2}{*}{90 per cent $\mathrm{OA}(\mathrm{DL})$} & Hyaline cartilage & Hyaline cartilage & $3.26 \mathrm{E}-06$ \\
\hline & Normal bone & Normal bone & $2.36 \mathrm{E}-09$ \\
\hline \multirow[t]{2}{*}{90 per cent $\mathrm{OA}+\mathrm{OP}(\mathrm{DL})$} & Hyaline cartilage & Hyaline cartilage & $3.26 \mathrm{E}-06$ \\
\hline & Osteoporotic bone & Osteoporotic bone & $1.65 \mathrm{E}-09$ \\
\hline 100 per cent $\mathrm{OA}+\mathrm{OP}(\mathrm{SL})$ & Osteoporotic bone & Osteoporotic bone & $1.65 \mathrm{E}-09$ \\
\hline Ti-UHMWPE (SL) & Titanium & UHMWPE & $2.34 \mathrm{E}-08$ \\
\hline
\end{tabular}

Two models include a double-contact layer (DL), being the remaining models composed by a single-contact layer (SL). The acronyms OA and OP indicate the diseases osteoarthritis and osteoporosis, respectively. The 90 per cent OA knee corresponds to a knee which had lost 90 per cent of the original cartilage layer.

Table 4 Mechanical properties of the contact materials: Young's modulus $(E)$ and Poisson's ratio $(v)$

\begin{tabular}{lrr}
\hline Material & $E(\mathrm{MPa})$ & $v$ \\
\hline Hyaline cartilage [67] & 24 & 0.38 \\
Normal bone [67] & 17200 & 0.39 \\
Osteoporotic bone [66] & 12000 & 0.39 \\
Titanium [68] & 113800 & 0.34 \\
UHMWPE [68] & 800 & 0.46 \\
\hline
\end{tabular}

properties. For example, OA is the most common cause of musculoskeletal pain and disability at the knee joint. In a simple way, OA can be defined as mechanically induced cartilage loss $[\mathbf{6 3}, 64]$, which is characterized by a decrease in cartilage volume and thickness that could ultimately lead to the exposition of the underlying bone. Nevertheless, OA diseases may be initiated by multiple factors, not only mechanical factors, but also biological, genetic, developmental, metabolic, and traumatic [64]. The knee OA entails not only cartilage loss, but also bony remodelling, with capsular stretching and weakness of the muscles that surround the knee joint. Localized areas of cartilage loss may lead to further cartilage loss by increasing the focal stress across the joint. With a large enough area of cartilage loss or with bony remodelling, the joint becomes tilted, and the malalignment develops, which is the most powerful risk factor for structural deterioration of the joint $[64,65]$. Therefore, in OA severe stage, the contact material started to be composed by the subchondral bone, whose mechanical behaviour is quite different from the cartilage. In a similar way, for patients with osteoporosis (OP), which is characterized by the lose of bone mechanical properties [66], the material properties can vary significantly. Thus, in order to examine how a healthy, a pathologic, and an artificial knee joint response is affected by the intrinsic material properties, different situations are considered. In particular, six different contact material conditions are utilized, namely one for the healthy knee model, three for pathologic knee models, and two for artificial knee models, as Table 3 summarizes. The mechanical properties necessary to characterize the different contact materials are listed in Table 4.

As mentioned above, OA initiation and its progression can have multiple precursors, such as mechanic, biologic, and genetic, among others. Since it is extremely difficult to develop a pathologic knee model that accounts for all these factors, a simplified model is developed. In this model, only the cartilage loss effect mechanically induced is considered, being the remaining OA factors neglected. To model the cartilage loss, a reduction on the original thickness of the cartilage layer is made. This reduction is considered proportional to the percent of OA severity. In order to simulate a pathologic knee which had lost 90 per cent of the original cartilage layer (90 per cent OA knee), some modelling adjustments have to be done. Therefore, the double-layer model is implemented. For the sake of simplicity, the first layer is modelled as a layer with uniform thickness that is located along the geometrical profile. Hence, the contact detection algorithm did not require any change and the contact law has to be adapted to the double-layer concept, according to

$$
F_{\mathrm{N}}= \begin{cases}F_{1} & \text { if } \delta \leq h_{1} \\ F_{1}^{\max }+F_{2} & \text { if } \delta>h_{1}\end{cases}
$$

where $F_{\mathrm{N}}$ is the total normal contact force, $F_{1}$ and $F_{1}^{\text {max }}$ the normal contact force resultant from a partial or total indentation of the thickness of the first contact layer, and $F_{2}$ the normal contact force at the second contact layer that is null when the relative indentation is smaller than or equal to the thickness of the first layer. For instance, for Hertz contact law, equation (20) can be written as

$$
F_{\mathrm{N}}= \begin{cases}K_{1} \delta^{n} & \text { if } \delta \leq h_{1} \\ K_{1} h_{1}^{n}+K_{2}\left(\delta-h_{1}\right)^{n} & \text { if } \delta>h_{1}\end{cases}
$$

where $K_{1}$ and $K_{2}$ represent the generalized stiffness parameters of first and second contact layers, respectively, having the remaining parameters with the same meaning as described above. 


\section{RESULTS AND DISCUSSION}

In this section, results obtained from computational simulations of the mathematical multibody knee presented in section 2 are used to analyse and compare the effect of the different variables on the knee dynamic response. The simulations are performed for a duration of $0.4 \mathrm{~s}$ of with a time step equal to $1 \mathrm{E}-4 \mathrm{~s}$. With the purpose to have a term of comparison, the knee model corresponding to spherical femoral condyle and flat tibial plateau, coated by hyaline cartilage, is used as reference model, being the contact forces evaluated by employing the Hertz contact law.

Since the main purpose of this research study is to perform a comparative study on the influence of the main contact factors that can affect the dynamic behaviour of the multibody knee model presented, the three parameters considered are the contact

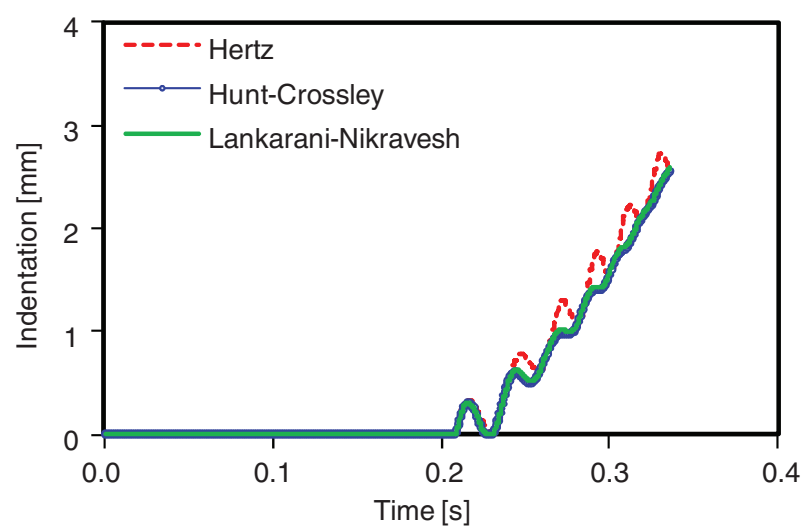

Fig. 5 Evolution of the indentation for the different contact force models, namely Hertz contact law, Hunt-Crossley model, and LankaraniNikravesh formulation. The simulations are performed considering the convex sphereplane geometrical model where the cartilage is the only contact material used

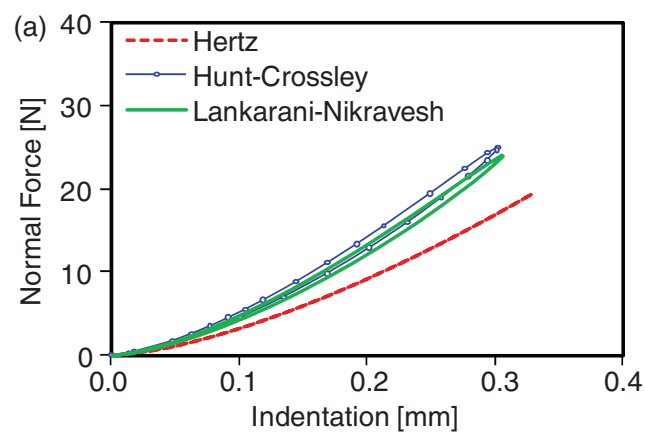

force model, contact geometric configuration, and material properties of the contacting surfaces. In this context, the system dynamic response is quantified by the plots of the indention and contact forces. Other important issues, such as muscles and ligaments actions are out of the scope of this study, interested readers can refer to references [2] and [37].

\subsection{Influence of the contact force model}

With the intention to assess the influence of the contact force model on the dynamic response of the multibody knee joint model, several computational simulations are performed using three different constitutive contact laws, namely the Hertz contact law, Hunt-Crossley model, and Lankarani-Nikravesh formulation. For the case of dissipative force models, the value of the coefficient of restitution of the hyaline cartilage is equal to 0.616 [69]. The dynamic behaviour of the knee joint model is quantified by plotting the values of the indentation and the magnitude of the contact force developed during contact, as illustrated in Figs 5 and 6, respectively.

Figure 5 shows the evolution of the indentation for the different contact force models, from which it can be observed that the use of Hertz contact law produces higher indentations. This fact is logical because the Hertz law is a pure elastic force model that does not account for any energy dissipation during the contact process. This observation is also visible in the diagram of Fig. 6, where the curve for the Hertz law does not present any hysteresis loop, meaning that the energy stored during the loading phase is exactly the same that is restored during the unloading phase. In turn, form the dissipative contact force models, Hunt and Crossley, and Lankarani and Nikravesh models, exhibit similar response, being the indentation evolution smoother over the dynamic simulation, as depicted in Fig. 5. The observation is

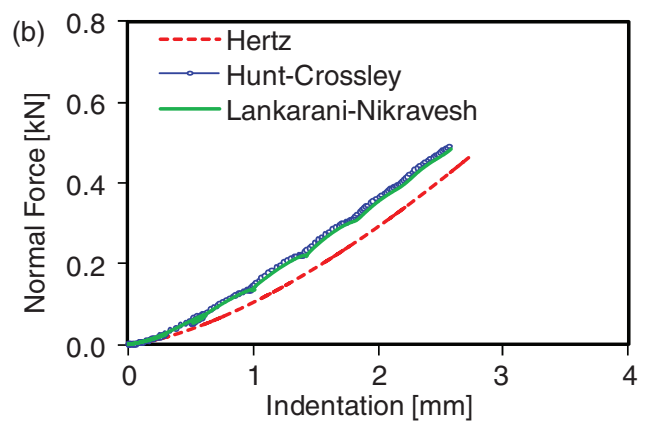

Fig. 6 Normal contact force versus indentation for the different contact force models: (a) first impact simulation and (b) continuous contact scenario. The simulations are performed considering the convex sphere-plane geometrical model, where the articular cartilage is the only contact material used 


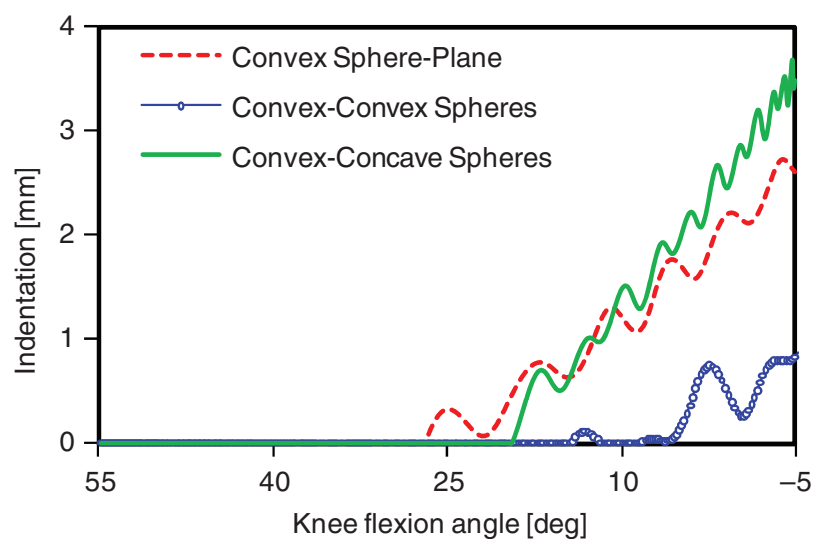

Fig. 7 Indentation versus knee flexion angle using different geometrical models. The simulations are performed using the Hertz contact law and considering the cartilage as the only contact material

clearly associated with the hysteresis loop that occurs for these two models, as it is shown in the plots of Fig. 6(a) relative to the first impact. Figure 6(a) illustrates the dissipative nature of the elastic force models where the complete loading and unloading phases are quite visible. Furthermore, it should be highlighted that the continuous contact scenario plotted in Fig. 6(b) does not present a closed loop, since the contacts are not complete, meaning that the unloading phase does not occur totally. This situation is also quite visible in the plots of Fig. 5.

\subsection{Influence of the contact geometrical conformality}

In order to study the influence of the geometrical conformality of the contact bodies on the dynamic behaviour of the knee joint, several computational simulations are carried out using the three contact geometrical approaches described in section 2.2, namely convex-convex sphere model, convex-concave sphere model, and convex sphere-plane model. Similar to the case presented in the previous section, the dynamic response of the knee joint model is quantified by studying the indentation (Fig. 7) and contact force (Fig. 8) plots.

By analysing Fig. 7, it can be observed that the conformality of the contact bodies has a significant influence on the global contact results, namely in terms of indentation and, ultimately, on the contact force. Figure 7 shows that the convex-concave sphere model exhibits the highest level of indentation. This observation is explained by the dynamic nature of the formulation used in this study, in which the contact force is an explicit function of the system configuration and the contact properties. In particular, the

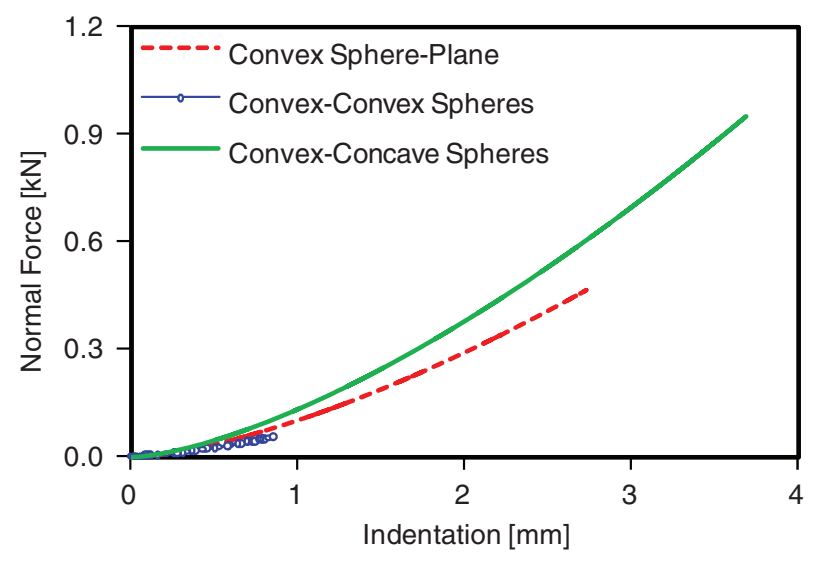

Fig. 8 Normal contact force versus indentation using different geometrical models. The simulations are performed using the Hertz contact law and considering the cartilage as the only contact material

higher radius of the medial femur $\left(R_{\mathrm{f}}=30.4 \mathrm{~mm}\right)$ compared with the radius of the lateral femur $\left(R_{\mathrm{f}}=22.0 \mathrm{~mm}\right)$ also contributes for this result. This outcome is also visible in the plots of Fig. 8, where the highest and the lowest contact forces correspond to the convex-concave sphere and convex-convex sphere models, respectively. These results can be used to understand the major incidence of OA at the medial compartment of the knee joint, which exhibits a conformal contact scenario in the anterior-posterior direction [49]. This idea is supported by this fact that the multibody knee model has been simulated for similar conditions. However, the dynamic behaviour of the actual knee can be affected by the presence of the surrounding structures, such as the menisci and muscle, which play an important role in the progression of the OA.

\subsection{Influence of the contact material properties}

In the simulations performed in this section, the influence of six contact material interfaces on the knee joint dynamics is analysed. Because the contact material properties vary, some simplifications are made with the purpose to keep the analysis simple. Thus, the Hertz law is the only contact force model considered, being the contact materials modelled with non-linear elastic behaviour. Furthermore, in the simulations performed, the viscoelastic properties of the hyaline cartilage and the ultra-high molecular weight polyethylene (UHMWPE), in particular the stress relaxation, are not taken into account. The obtained results are organized in two groups: the knee models with cartilage (healthy knee, 90 per cent OA knee and 90 per cent OA + OP knee) and the knee models without cartilage (100 per cent OA + OP 


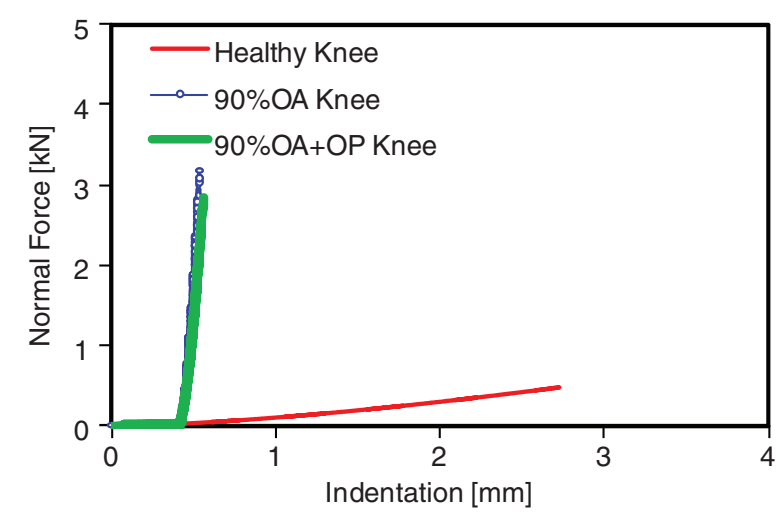

Fig. 9 Normal contact force versus indentation using different knee models with cartilage. The simulations are performed using the Hertz contact law and considering the convex sphere-plane geometrical model

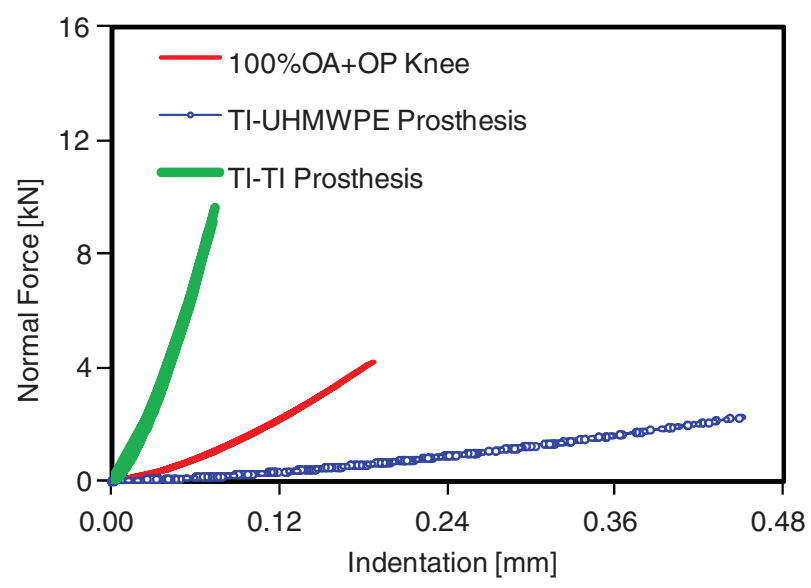

Fig. 10 Normal contact force versus indentation using different knee models without cartilage. The simulations are performed using the Hertz contact law and considering the convex sphere-plane geometrical model

knee, Ti-UHMWPE prosthesis, and Ti-Ti prosthesis). Figure 9 depicts the results of the three knee models for the following cartilage properties: the healthy knee, the 90 per cent OA knee, and the 90 per cent $\mathrm{OA}+\mathrm{OP}$ knee. From the analysis of the plots of Fig. 9, it can be drawn that the knee joint models exhibit the same response for indentation up to $0.415 \mathrm{~mm}$, which corresponds to a 10 per cent of the original thickness of the hyaline cartilage. Nevertheless, when the indentation is greater than $0.415 \mathrm{~mm}$, the contact forces developed at the pathological knees significantly increase. This situation is clearly associated with the contact material, changing from cartilage to bone, being the latter a stiffer material. Analysing the two pathological knees, it can be concluded that they present similar behaviour. Although it can be observed that, for the same indentation, the 90 per cent OA knee produces higher normal contact forces

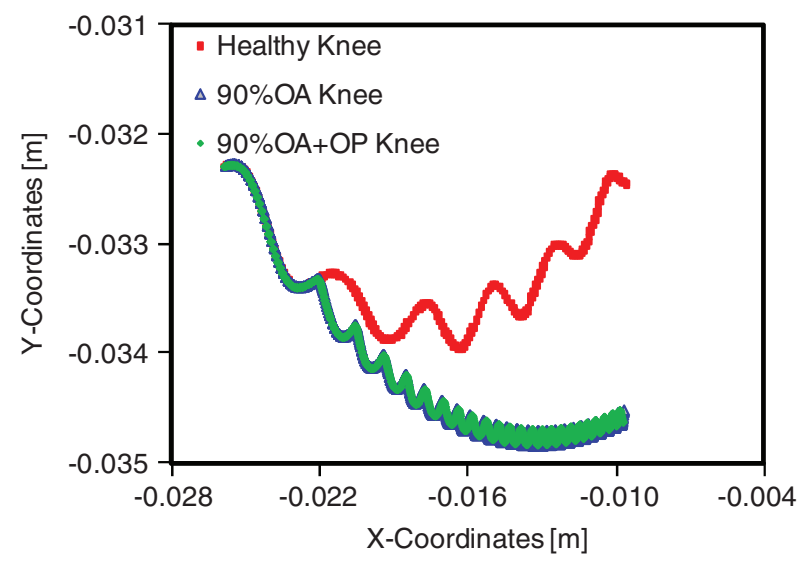

Fig. 11 Tibia contact point trajectories using different knee models with cartilage. The simulations are performed using the Hertz contact law and considering the convex sphere-plane geometrical model

when compared to the 90 per cent OA + OP knee case. This outcome, also reported by Dickenson et al. [66], is reasonable since the OP is a metabolic disease characterized by a general reduction in bone mass, which results in a lower stiffness and, consequently, reduces the level of the normal contact forces [66]. Figure 10 includes the results for the knee joint modelled without cartilage, namely the 100 per cent OA + OP knee, the Ti-UHMWPE prosthesis, and the Ti-Ti prosthesis. By comparing the plots of Figs 9 and 10, it can be drawn that the absence of cartilage leads to higher contact forces even with smaller indentations. This observation highlights the key role played by the cartilage as shock absorber and load spreader.

As far as the artificial knee models are concerned, it should be noticed that the Ti-UHMWPE prosthesis (Fig. 10) presents a dynamic response closer to the case of the healthy knee (Fig. 9). Hence, it can be expected that the Ti-UHMWPE prosthesis would have a higher performance than the Ti-Ti prosthesis. This result supports the idea that the UHMWPE is the preferred choice by the surgeons, since there are no currently acceptable alternatives, clinically proven, able to overcome its performance as bearing material in knee [68].

Figures 11 and 12 illustrate the tibia contact point trajectories from the knee models with cartilage and without cartilage, respectively. By comparing plot of the healthy knee (Fig. 11) with those obtained for the knee models without cartilage (Fig. 12), it can be concluded that the evolution of the tibia contact point trajectory is clearly affected by the properties of the contact materials.

At this stage, it is important to note that the results reported here, for the case in which the knee is modelled as Ti-UHMWPE prosthesis, are corroborated by 


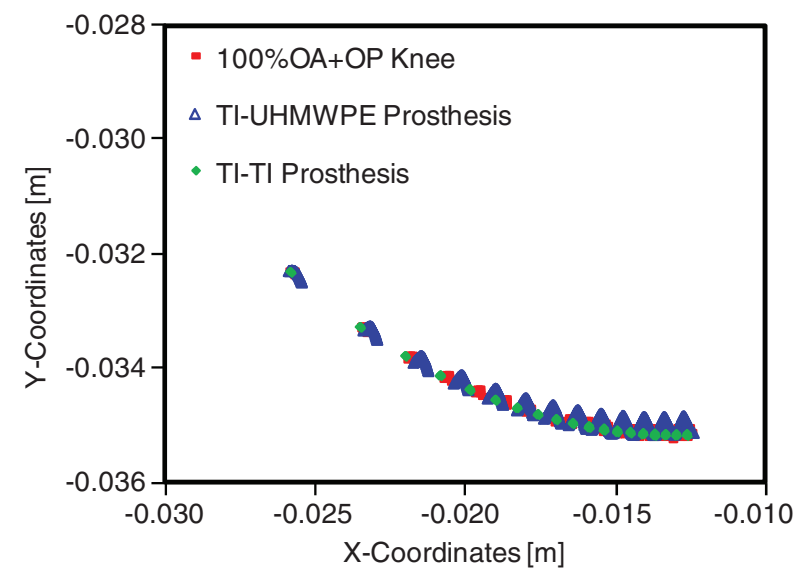

Fig. 12 Tibia contact point trajectories using different knee models without cartilage. The simulations are performed using the Hertz contact law and considering the convex sphere-plane geometrical model

those published by Pérez-González et al. [19]. In the investigations carried out by Pérez-González et al., the Hertz law was used to evaluate the contact forces, as it is case of presented in this section.

\section{CONCLUDING REMARKS}

In this study, the influence of the contact model on the dynamic response of the human knee joint has been studied. The three contact parameters considered are: (a) the constitutive contact force law, (b) the geometrical description of the contacting bodies, and (c) the material properties of the contact pair.

As far as the contact force model is concerned, it was observed that the dissipative contact force laws, namely Hunt-Crossley and Lankarani-Nikravesh models, are more appropriate than the pure Hertz law to describe the dynamic response of the knee joint. These models, besides to express the non-linear behaviour of cartilage, also take into account its viscoelastic nature, which provides shock absorption and, consequently, the energy dissipation during the impact [12].

The results obtained for different contact geometries show that the knee medial compartment, which has a conformal configuration, presents higher contact forces when compared with the knee lateral compartment. This observation can, in some measure, explain the major incidence of $\mathrm{OA}$ in the knee medial compartment [49]. Nonetheless, it should be highlighted that other relevant parameters, neglected in the present model, may also contribute to the knee OA evolution, such as menisci and muscles. In addition, the general gait parameters (stride length, cadence, etc.) and daily activities, such as labour tasks and sport practice, can also influence the knee joint dynamic response [70].
In order to assess the influence of the contact material properties on the dynamic response of the multibody knee joint model, a healthy knee model was compared to three different pathologic cases and two artificial knee models. The results showed that the cartilage reduces the contact force experienced by the models without cartilage, and extends the period of contact. This cartilage role is of paramount importance during walking, since the ground reaction force typically rises to a peak after heel strikes and during this phase, loads across the knee joint have been calculated to be about three times the body weight $[\mathbf{7 0}]$. Moreover, the computational simulations showed that the presence of OP does not change the level of contact forces at the OA knee model, meaning that this disease does not contribute to the OA progression. Regarding the artificial knees, the results showed that Ti-UHMWPE prosthesis exhibits higher performance when compared to the Ti-Ti solution, which supports the idea of the surgeons in excluding the metal-metal interface for the TKRs [68].

\section{FUNDING}

The research work presented in this article was supported by The Portuguese Foundation for Science and Technology (FCT) [grant no. SFRH/BD/40164/ 2007] and through the projects PROPAFE [PTDC/ EMEPME/67687/2006], DACHOR [MIT-Pt/ BSHHMS/0042/2008], and BIOJOINTS [PTDC/EMEPME/099764/2008].

\section{ACKNOWLEDGEMENTS}

The authors thank the Portuguese Foundation for Science and Technology (FCT) for the support given through the projects mentioned above. The first author expresses her gratitude to FCT for the PhD grant no. SFRH/BD/40164/2007.

(C) Authors 2011

\section{REFERENCES}

1 Flores, P. and Ambrosio, J. On the contact detection for contact-impact analysis in multibody systems. Multibody Syst. Dyn., 2010, 24, 103-122.

2 Machado, M., Flores, P., Claro, J. C. P., Ambrósio, J., Silva, M. T., Completo, A., and Lankarani, H. M. Development of a planar multibody model of the human knee joint. Nonlinear Dyn., 2010, 60, 459-478.

3 Flores, P., Ambrósio, J., Claro, J. C. P., and Lankarani, H. M. Influence of the contact-impact force model on the dynamic response of multi-body systems. Proc. IMechE, Part K: J. Multi-body Dynamics, 2006, 220, 21-34. 
4 Lopes, D. S., Silva, M. T., Ambrósio, J., and Flores, P. A mathematical framework for rigid contact detection between quadric and superquadric surfaces. Multibody Sys. Dyn., 2010, 24, 255-280.

5 Delp, S. L. and Loan, J. P. A graphics-based software system to develop and analyze models of musculoskeletal structures. Comput. Biol. Med., 1995, 25, 21-34.

6 Shuxian, Z., Wanhua, Z., and Bingheng, L. 3D reconstruction of the structure of a residual limb for customising the design of a prosthetic socket. Med. Eng. Phys., 2005, 27, 67-74.

7 Silva, P., Silva, M. T., and Martins, J. M. Evaluation of the contact forces developed in the lower limb/ orthosis interface for comfort design. Multibody Syst. Dyn., 2010, 24, 367-388.

8 Dopico, D., Luaces, A., Gonzalez, M., and Cuadrado, J. Dealing with multiple contacts in a human-in-the-loop-application. Multibody Syst. Dyn., 2010, 25, 167-183.

9 Hippmann, G. An algorithm for compliant contact between complexity shaped bodies. Multibody Syst. Dyn., 2004, 12, 345-362.

10 Lin M. C. and Gottschalk S. Collision detection between geometric models: a survey. In Proceedings of the 8th IMA Conference on Mathematics of surfaces (Ed. R. Cripps), Birmingham, UK, 31 August-2 September, 1998, pp. 37-56.

11 Choi, J., Ryu, H. S., Kim, C. W., and Choi, J. H. An efficient and robust contact algorithm for a compliant contact force model between bodies of complex geometry. Multibody Syst. Dyn., 2010, 23, 99-120.

12 Gilardi, G. and Sharf, I. Literature survey of contact dynamics modelling. Mech. Mach. Theory, 2002, 37, 1213-1239.

13 Flores, P., Machado, M., Silva, M. T., and Martins, J. M. On the continuous contact force models for soft materials in multibody dynamics. Multibody Syst. Dyn., 2011, 25, 357-375.

14 Tasora, A., Negrut, D., and Anitescu, M. Large-scale parallel multi-body dynamics with frictional contact on the graphical processing unit. Proc. IMechE, Part K: J. Multi-body Dynamics, 2008, 222, 315-326.

15 Flores, P., Lankarani, H. M., Ambrósio, J., and Claro, J. C. P. Modelling lubricated revolute joints in multibody mechanical systems. Proc. IMechE, Part K: J. Multi-body Dynamics, 2004, 218, 183-190.

16 Flores, P., Ambrósio, J., Claro, J. C. P., and Lankarani, H. M. Dynamic behaviour of planar rigid multibody systems including revolute joints with clearance. Proc. IMechE, Part K: J. Multi-body Dynamics, 2007, 221, 161-174.

17 Flores, P. Modeling and simulation of wear in revolute clearance joints in multibody systems. Mech. Mach. Theory, 2009, 44, 1211-1222.

18 Mukras, S., Kim, N. H., Mauntler, N. A., Schmitz, T., and Sawyer, W. G. Comparison between elastic foundation contact force models in wear analysis of planar multibody system. J. Tribol., 2010, 132, 0316041-031604.11.

19 Pérez-González, A., Fenollosa-Esteve, C., SanchoBru, J. L., Sánchez-Marín, F. T., Vergara, M., and
Rodríguez-Cervantes, P. J. A modified elastic foundation contact model for application in 3D models of the prosthetic knee. Med. Eng. Phys., 2008, 30, 387-398.

20 Hirokawa, S. Biomechanics of the knee joint: a critical review. Crit. Rev. Biomed. Eng., 1993, 21, 79-135.

21 Wismans, J., Veldpaus, F., and Janssen, J. A Threedimensional mathematical model of the knee joint. J. Biomech., 1980, 13, 677-685.

22 Moeinzadeh, M. H. Two-dimensional dynamic modelling of human knee joint. J. Biomech., 1983, 16, 253-264.

23 Engin, A. E. and Tumer, S. T. Improved dynamic model of the human knee joint and its response to impact loading on the lower leg. J. Biomech. Eng., 1993, 115, 137-143.

24 Ling, Z.-K., Guo, H.-Q., and Boersma, S. Analytical study on the kinematic and dynamic behaviors of a knee joint. Med. Eng. Phys., 1997, 19, 29-36.

25 Abdel-Rahman, E. and Hefzy, M. S. A two-dimensional dynamic anatomical model of the human knee joint. J. Biomech. Eng., 1993, 115, 357-365.

26 Abdel-Rahman, E. and Hefzy, M. S. Three-dimensional dynamic behaviour of the human knee joint under impact loading. Med. Eng. Phys., 1998, 20, 276-290.

27 Hirokawa, S. Three-dimensional mathematical model analysis of the patellofemoral joint. $J$. Biomech., 1991, 24, 659-671.

28 Blankevoort, L., Kuiper, J. H., Huiskes, R., and Grootenboer, H. J. Articular contact in a threedimensional model of the knee. J. Biomech., 1991, 24, 1019-1031.

29 Kalker, J. J. Three-dimensional elastic bodies in rolling contact, 1990 (Kluwer Academic Publishers, Dordrecht).

30 Blankevoort, L. and Huiskes, R. Validation of a three-dimensional model of the knee. J. Biomech., 1996, 29, 955-961.

31 Gill, H. S. and O'Connor, J. J. Biarticulating two-dimensional computer model of the human patellofemoral joint. Clin. Biomech., 1996, 11, 81-89.

32 Chittajallu, S. K. and Kohrt, K. G. FORM2D-A mathematical model of the knee. Math. Comput. Modell., 1996, 24, 91-101.

33 Bendjaballah, M. Z., Shirazi-Adl, A., and Zukor, D. J. Biomechanics of the human knee joint in compression: reconstruction, mesh generation and finite element analysis. Knee, 1995, 2, 69-79.

34 Heegaard, J., Leyvraz, P. F., Curnier, A., Rakotomanana, L., and Huiskes, R. The Biomechanics of the human patella during passive knee flexion. J. Biomech., 1995, 28, 1265-1279.

35 Sathasivam, S. and Walker, P. S. A Computer model with surface friction for the prediction of the total knee kinematics. J. Biomech., 1997, 30, 177-184.

36 Li, G., Sakamoto, M., and Chao, E. A Comparison of different methods in predicting static pressure distribution in articulating joints. J. Biomech., 1997, 30, 635-638. 
37 Pandy, M. G., Sasaki, K., and Kim, S. A Threedimensional musculoskeletal model of the human knee joint. Part 1: theoretical construction. Comput. Meth. Biomech. Biomed. Eng., 1997, 1, 87-108.

38 Zhu, Y., Chen, J. X., Xiao, S., and MacMahon, E. 3D knee modeling and biomechanical simulation. Comput. Sci. Eng., 1999, 1, 82-87.

39 Kwak, S. D., Blankevoort, L., and Ateshian, G. A. A mathematical formulation for 3D quasi-static multibody models of diarthrodial joints. Comput. Meth. Biomech. Biomed. Eng., 2000, 3, 41-64.

40 Piazza, S. J. and Delp, S. L. Simulation of total knee replacement motion during a step-up task. J. Biomech. Eng., 2001, 123, 599-606.

41 Li, G., Lopez, O., and Rubash, H. Variability of a three-dimensional finite element model constructed using magnetic resonance images of a knee for joint contact stress analysis. J. Biomech. Eng., 2001, 123, 341-346.

42 Dhaler, Y. Y. and Kahn, L. E. The effect of vastus medialis forces on patello-femoral contact: a model-based study. J. Biomech. Eng., 2002, 124, 758-767.

43 Caruntu, D. I. and Hefzy, M. S. 3-D Anatomically based dynamic modeling of the human knee to include tibio-femoral and patello-femoral Joints. $J$. Biomech. Eng., 2004, 126, 44-53.

44 Bei, Y. and Fregly, B. J. Multibody dynamic simulation of the knee contact mechanics. Med. Eng. Phys., 2004, 26, 777-789.

45 Besier, T. F., Gold, G. E., Beaupre, G. S., and Delp, S. L. A Modeling framework to estimate patellofemoral joint cartilage stress in vivo. Med. Sci. Sports Exerc., 2005, 37, 1924-1930.

46 Fernandez, J. W. and Hunter, P. J. An anatomically based patient-specific finite element model of patella articulation: towards a diagnostic tool. Biomech. Model. Mechanobiol., 2005, 4, 20-38.

47 Han, S.-K., Federico, S., Epstein, M., and Herzog, W. An articular cartilage contact model based on real surface geometry. J. Biomech., 2005, 38, 179-184.

48 Halloran, J. P., Easley, S. K., Petrella, A. J., and Rullkoetter, P. J. Comparison of deformable and elastic foundation finite element simulations for predicting knee replacement mechanics. J. Biomech. Eng., 2005, 127, 813-818.

49 Koo, S. and Andriacchi, T. P. A comparison of the influence of global functional loads vs. local contact anatomy on articular cartilage thickness at the knee. J. Biomech. 2007, 40, 2961-2966.

50 Lin, Y. C., Haftka, R. T., Queipo, N. V., and Fregly, B. J. Surrogate articular contact models for computationally efficient multibody dynamic simulations. Med. Eng. Phys., 2010, 32, 584-594.

51 Hertz, H. On the contact of solids - on the contact of rigid elastic solids and on hardness. In Miscellaneous papers (Eds H. Hertz, D. E. Jones, and G. A. Schott), 1896, pp. 146-183 (Macmillan, London).

52 Hunt, K. H. and Crossley, F. R. Coefficient of restitution interpreted as damping in vibroimpact. J. Appl. Mech., 1975, 42, 440-445.
53 Lankarani, H. M. and Nikravesh, P. E. A contact force model with hysteresis damping for impact analysis of multibody systems. J. Mech. Des., 1990, 112, 369-376.

54 Nikravesh, P. E. Newtonian-based methodologies in multi-body dynamics. Proc. IMechE, Part K: J. Multibody Dynamics, 2008, 222, 277-288.

55 Orlandea, N. V. From Newtonian dynamics to sparse tableaux formulation and multi-body dynamics. Proc. IMechE, Part K: J. Multi-body Dynamics, 2008, 222, 301-314.

56 Schmitke, C., Morency, K., and McPhee, J. Using graph theory and symbolic computing to generate efficient models for multi-body vehicle dynamics. Proc. IMechE, Part K: J. Multi-body Dynamics, 2008, 222, 339-352.

57 Lugrís, U., Naya, M. A., Luaces, A., and Cuadrado, J. Efficient calculation of the inertia terms in floating frame of reference formulations for flexible multibody dynamics. Proc. IMechE, Part K: J. Multi-body Dynamics, 2009, 223, 147-157.

58 Engin, A. E. and Akkas, N. Application of a fluidfilled spherical sandwich shell as a biodynamic head injury model for primates. Aviat. Space Environ. Med., 1978, 49, 120-124.

59 Adams, G. G. and Nosonovsky, M. Contact modeling - forces. Tribol. Int., 2000, 33, 431-442.

60 Liu, C.-S., Zhang, K., and Yang, L. Normal forcedisplacement relationship of spherical joints with clearances. J. Comput. Nonlinear Dyn., 2006, 1, 161-167.

61 Koo, S., Gold, G., and Andriacchi, T. P. Considerations in measuring cartilage thickness using MRI: factors influencing reproducibility and accuracy. Osteoarthr. Cartilage, 2005, 13, 782-789.

62 Martelli, S., Pinskerova, V., and Visani, A. Anatomical investigation on the knee by means of computer-dissection. J. Mech. Med. Biol., 2006, 6, 55-73.

63 Wilson, W., van Donkelaar, C. C., van Rietbergen, R., and Huiskes, R. The role of computational models in the search for the mechanical behavior and damage mechanisms of articular cartilage. Med. Eng. Phys., 2005, 27, 810-826.

64 Moskowitz, R. W. Osteoarthritis: diagnosis and medical/surgical management, 3 edition, 2007 (Lippincott Williams \& Wilkins, Philadelphia, Pennsylvania).

65 Felson, D. T. Osteoarthritis of the knee. N. Engl. J. Med., 2006, 354, 841-848.

66 Dickenson, R. P., Hutton, W. C., and Stott, J. R. The mechanical properties of bone in osteoporosis. $J$. Bone Joint Surg. Br., 1981, 63B, 233-238.

67 Herman, I. P. Physics of the human body, 2007 (Springer-Verlag, New York).

68 Kurtz, S. M. The UHMWPE handbook-principles and clinical applications in total joint replacement, 2004 (Elsevier Academic Press, San Diego, California).

69 Burgin, L. V. and Aspen, R. M. Impact testing to determine the mechanical properties of articular cartilage in isolation and on bone. J. Mater. Sci. Mater. Med., 2008, 19, 703-711.

70 Andriacchi, T. and Dyrby, C. Gait analysis and total knee replacement. In Total knee arthroplasty - a 
guide to get better performance (Eds J. Bellemans, M. D. Ries, and J. Victor), 2005, pp. 38-42, (Springer, Berlin/Heidelberg).

\section{APPENDIX}

\section{Notation}

$A \quad$ amplitude of the external applied force

$A_{j}, B_{j} \quad$ border points of the contact plane $j$

AB vector that connects the point $A_{j}$ to the point $B_{j}$

AC vector that connects the point $A_{j}$ to the point $C_{i}$

$c_{r} \quad$ restitution coefficient

$C_{i, j} \quad$ centre of a sphere $i, j$

$d$ magnitude of distance vector

d distance vector

$D \quad$ damping coefficient

$E_{\mathrm{f}} \quad$ Young's modulus of the femur contact material

$E_{\mathrm{t}} \quad$ Young's modulus of the tibia contact material

$F_{\mathrm{e}} \quad$ external applied force at the centre of mass of the tibia

$F_{1} \quad$ knee ligament force

$F_{\mathrm{N}} \quad$ normal contact force

$F_{1} \quad$ normal contact force resultant from a partial indentation of the thickness of the first contact layer

$F_{2} \quad$ normal contact force at the second contact layer

$F_{1}^{\max }$ normal contact force resultant from a total indentation of the thickness of the first contact layer $h_{1} \quad$ thickness of the first contact layer

$i, j \quad$ pair of contact bodies

$k_{l} \quad$ knee ligament stiffness

$K \quad$ generalized stiffness parameter

$K_{1} \quad$ generalized stiffness parameter of first contact layer

$K_{2} \quad$ generalized stiffness parameter of second contact layer

$l_{1} \quad$ knee ligament current length

$l_{1}^{0} \quad$ knee ligament unstrained length

$n$ non-linearity exponent

$\mathbf{r}_{i}^{C_{i}} \quad$ global coordinate vector of centre point $C_{i}$

$\mathbf{r}_{j}^{C_{j}} \quad$ global coordinate vector of centre point $C_{j}$

$R_{i, j} \quad$ radius of body $i, j$

$t \quad$ time variable

$t_{\mathrm{d}} \quad$ time duration

$X Y \quad$ global coordinate system

$\delta \quad$ relative indentation

$\dot{\delta} \quad$ relative normal indentation velocity

$\dot{\delta}^{0} \quad$ initial relative normal indentation velocity

$\theta_{A} \quad$ angle between vectors $\mathbf{A C}$ and $\mathbf{A B}$

$v_{\mathrm{f}} \quad$ Poisson's ratio of the femur contact material

$v_{\mathrm{t}} \quad$ Poisson's ratio of the tibia contact material

$\xi \eta \quad$ body-fixed coordinate system

$\phi_{i} \quad$ rotation angle of the $\xi \eta$ body-fixed frame of body $i$ relative to the $X Y$ global axes

$\phi_{j} \quad$ rotation angle of the $\xi \eta$ body-fixed frame of body $j$ relative to the $X Y$ global axes

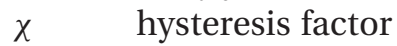

